УДК681.7.068/.069

\author{
М.И. Скворцов ${ }^{1,2}$, С.Р. Абдуллина ${ }^{1}$, А.А. Власов ${ }^{1}$ \\ ${ }^{1}$ Институт автоматики и электрометрии СО РАН, Новосибирск, Россия \\ ${ }^{2}$ Новосибирский национальный исследовательский государственный \\ университет, Новосибирск, Россия
}

\title{
ОПТИМИЗАЦИЯ ВОЛОКОННОГО ВКР-ЛАЗЕРА НА ОСНОВЕ МАССИВА ВОЛОКОННЫХ БРЭГГОВСКИХ РЕШЕТОК
}

\begin{abstract}
В работе исследована возможность оптимизации длины ВКР-лазера со случайной распределенной обратной связью, реализованной за счет записи массива волоконных брэгговских решеток (ВБР) со случайным набором фаз и амплитуд в пассивном волокне с сохранением поляризации. В результате была идентифицирована и удалена часть массива, вносившая большие потери, при этом длина массива ВБР уменьшилась с 13 до 6,7 м, а количество решеток в массиве - с 57 до 30. В оптимизированной конфигурации пороговая мощность накачки составляет менее $1 \mathrm{BT}$, а генерируемая мощность непрерывного линейно-поляризованного излучения на длине волны 1092 нм составляет около 3 Вт с каждого конца массива при мощности накачки 14 Вт. Ширина линии генерации при максимальной мощности составляет около 80 пм, а в припороговом режиме наблюдается одночастотная генерация с шириной $<50$ кГц.

Ключевые слова: волоконный лазер, ВКР-лазер, случайная распределенная обратная связь, волоконная брэгговская решетка.
\end{abstract}

\author{
M.I. Skvortsov ${ }^{1,2}$, S.R. Abdullina1, A.A. Vlasov ${ }^{1}$ \\ ${ }^{1}$ Institute of Automation and Electrometry, Siberian Branch of the Russian \\ Academy of Sciences, Novosibirsk, Russian Federation \\ ${ }^{2}$ Novosibirsk State University, Novosibirsk, Russian Federation \\ OPTIMIZATION OF FIBER RAMAN LASER BASED \\ ON FIBER BRAGG GRATINGS ARRAY
}

Optimization opportunities of random Raman fiber laser based onarray offiber Bragg gratings (FBGs) with random phases and amplitudes areinvestigated.Asa result, the part introducing large losses has been revealed and removed, at that the FNG array length has been reduced from 13 to $6.7 \mathrm{~m}$, and FBG number from 57 to 30 . In the optimized configuration the threshold pump power is below $1 \mathrm{~W}$, and generated linearly-polarized CW power at $1092 \mathrm{~nm}$ amounts to about $3 \mathrm{~W}$ from each end at pump power of $14 \mathrm{~W}$. The generation linewidth at maximum power amounts to $80 \mathrm{pm}$, while near threshold the laser exhibits single-frequency operation of $<50 \mathrm{kHz}$ width.

Keywords: fiber laser, Raman laser, random distributed feedback, fiber Bragg grating. 


\section{Введение}

Случайная распределенная обратная связь (СРОС) широко используется при создании волоконных лазеров. Можно выделить два типа СРОС: с некогерентным и когерентным механизмом обратной связи. Первый тип возникает в световоде из-за рэлеевского рассеяния на естественных флуктуациях показателя преломления и широко используется при создании ВКР-лазеров, при этом длина лазера составляет километры $[1,2]$. Второй тип СРОС реализуется за счет искусственных модуляций показателя преломления, таких как волоконная брэгговская решетка (ВБР) со случайными фазовыми сдвигами или массив ВБР, записанных через интервалы случайной длины [3-6]. Данный тип СРОС используется как в активных лазерах [3-5], так и в ВКР-лазерах [6], при этом применение случайных решеток позволяет значительно уменьшить длину резонатора и получить узкополосную генерацию вплоть до одночастотного режима. Первая реализация СРОС ВКР-лазера, генерирующего в области 1,5 мкм, со случайной ВБР длиной 1 м, содержащей 1500 фазовых сдвигов [6], продемонстрировала возможность получения узкополосного излучения вблизи порога генерации (с шириной линии 430 кГц), но максимальная мощность $(15$ мВт) и эффективность $(0,8 \%)$ такого лазера были очень низкими. Кроме того, изготовление подобной структуры требует специальной методики. В работе [7] предложена и реализована схема, основанная на массиве случайных ВБР, записанном в 13-метровом участке пассивного волокна с сохранением поляризации Fujikura SM98-PS-U25D (при общей длине волокна около 17 м). Массив состоял из $N=57$ ВБР длиной 4 см с одинаковой брэгговской длиной волны $\sim 1092,3$ нм, но различными фазами и амплитудами, записанными при помощи схемы записи с фазовой маской. Расстояние между смежными решетками составляло около 19 см при точности позиционирования 1 мм, вследствие чего между решетками формировался случайный фазовый сдвиг. Экспозиция в процессе записи ВБР определялась скоростью транслятора, которая задавалась генератором случайных чисел так, что коэффициент отражения решеток варьировался в диапазоне 10-15\%. В работе получены более высокая эффективность (34\%) и выходная мощность (5,7 Вт) по сравнению с результатами, продемонстрированными в [6], что стало возможным благодаря увеличению эффективной длины случайного лазера. Ширина линии генерации в припороговом режиме составила менее 100 кГц при мощности генерации 
12,5 мВт, а при максимальной мощности не превышала 80 пм, что гораздо меньше, чем в [6]. В реализованном лазере был зарегистрирован высокий уровень потерь как для излучения накачки, так и для генерации (до $35 \%$ ). При помощи оптического фильтра, пропускающего излучение с длиной волны $>1090$ нм, и визуализационной системы, регистрирующей излучение на длине волны около 1 мкм, было выявлено, что лазерная генерация осуществляется лишь на части массива ВБР.

В данной работе представлена экспериментальная оптимизация длины реализованного в [7] СРОС ВКР-лазера.

\section{Эксперимент}

Схема эксперимента представлена на рис. 1. В качестве источника накачки использовался непрерывный линейно-поляризованный лазер с длиной волны генерации 1045,2 нм мощностью до 14 Вт. В целях избавления от неконтролируемых потерь длина массива ВБР уменьшалась с сопутствующим контролем изменения уровня потерь.

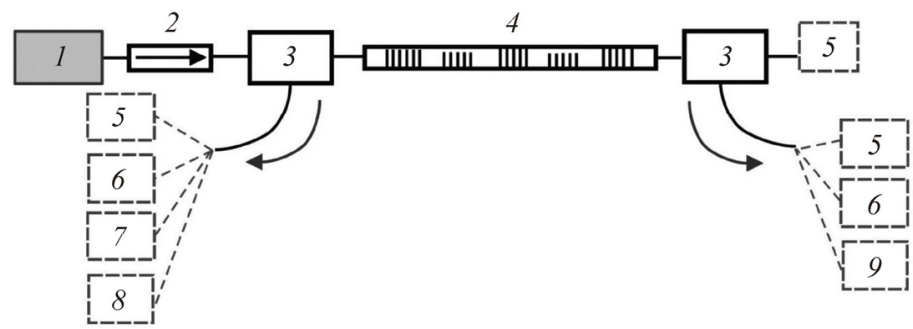

Рис. 1. Схема характеризации СРОС ВКР-лазера: 1 - излучение накачки; 2 - изолятор; 3 - спектрально-селективный разветвитель; 4 - массив ВБР; 5 - измеритель мощности; 6 - оптический спектроанализатор; 7 - осциллограф; 8 - поляриметр; 9 - радиочастотный анализатор

На рис. 2 приведены спектры пропускания массива ВБР начальной (конфигурация $1, N=57$ ) и конечной длины 6,7 м (конфигурация 2 , $N=30)$ на длине волны около 1092 нм. При уменьшении длины массива уровни потерь на длине волны излучения накачки и генерации снижались и достигли значений 3,4 и 3,9 \% соответственно. При этом пороговое значение мощности накачки также уменьшалось, в то время как для ВКР-лазера со СРОС, свободного от неконтролируемых потерь, уменьшение его длины должно приводить к росту пороговой мощности. Пороговая мощность накачки ВКР-лазера в процессе оптимизации его длины снизилась с 1,3 [7] до 0,84 Вт (рис. 3, б). 


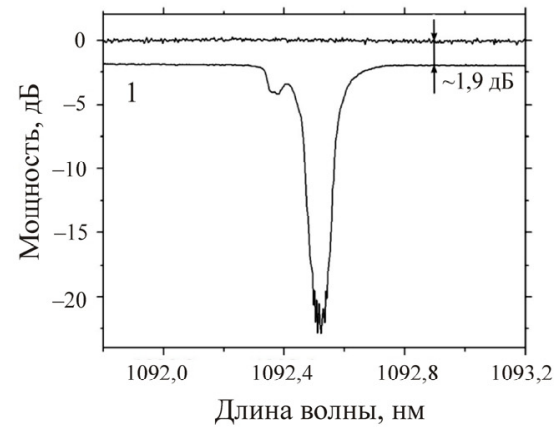

$a$

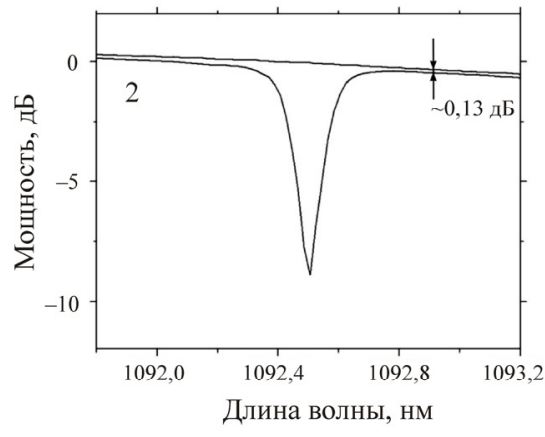

б

Рис. 2. Спектры пропускания массива ВБР для конфигурации $1(N=57)(a)$; для конфигурации $2(N=30)(б)$

На рис. 3 представлены мощности излучения прошедшей накачки и мощности стоксовых компонент, распространяющихся назад, в зависимости от мощности входящей накачки для конфигураций $1, a$ и $2, \sigma$. В конфигурации 2 мощности стоксовых компонент, распространяющихся вперед и назад, с хорошей точностью совпадают. Для обеих конфигураций можно выделить два режима генерации, которые характеризуются различными наклонами мощностных зависимостей для стоксовых компонент. Точка перегиба на графике для конфигурации 2 ( 3 Вт) (см. рис. 3,6 ) примерно соответствует оценочному значению пороговой мощности, полученному по формуле равенства потерь усилению.

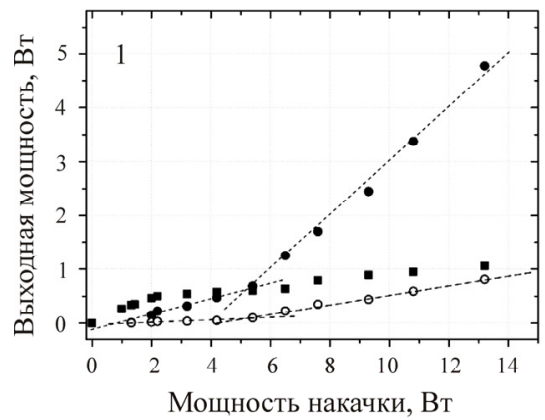

$a$

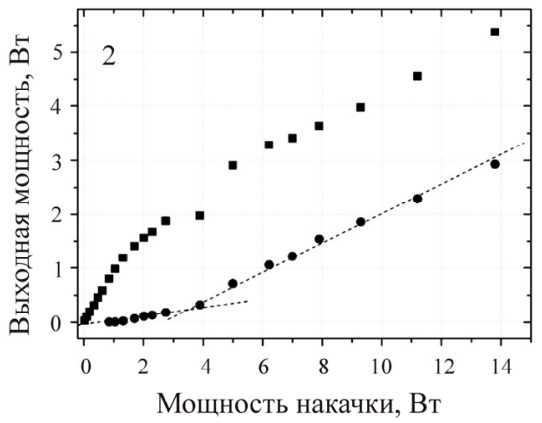

$\sigma$

Рис. 3. Мощность излучения накачки, прошедшего через массив ВБР (匹) и стоксовых компонент, распространяющихся вперед (о) и назад (•) в зависимости от входной мощности накачки для конфигураций 1 (a) и 2 (б)

Выходная мощность генерации при максимальной мощности накачки (14 Вт) составила около 3 Вт как для волны, распространяющейся сонаправленно с излучением накачки (вперед), так и для волны, рас- 
пространяющейся в противоположном направлении (назад). По сравнению с конфигурацией 1 кроме уменьшения порога изменилось соотношение мощностей, при этом суммарная выходная мощность сравнима с полученной в конфигурации 1 (около 6 Вт). При оптимизации длины резонатора уменьшается значение резонансных потерь с 1,9 дБ в старой конфигурации до 0,13 дБ в новой (см. рис. 2), что объясняет увеличение мощности стоксовой генерации, распространяющейся сонаправленно с накачкой. Уменьшение длины приводит к снижению мощности стоксовой генерации, распространяющейся в противоположном направлении, ввиду уменьшения расстояния прохождения в усиливающей среде. Можно утверждать, что после оптимизации резонатора СРОС-лазера эффективный фазовый сдвиг находится примерно по центру структуры, что является причиной симметричного перераспределения мощности генерации. В отличие от СРОС ВКР-лазеров на рэлеевском рассеянии [1] мощность прошедшей накачки после достижения порога продолжает расти.

На рис. 4, а показаны спектры стоксовой компоненты, распространяющейся назад, для конфигурации 1 и конфигурации 2 при близких значениях выходной мощности. Спектры разнесены по мощности для наглядности: как видно, при уменьшении количества ВБР в массиве в спектре генерации пропадает двухпиковая структура. Ширина линии стоксовой компоненты, распространяющейся назад, при максимальной выходной мощности не превышала 80 пм для, аналогично [7].

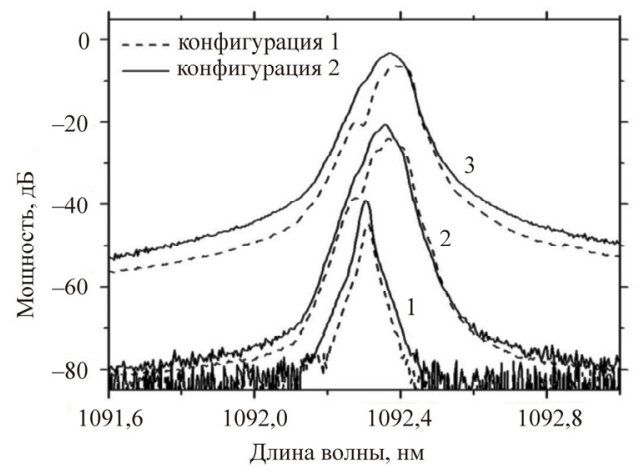

$a$

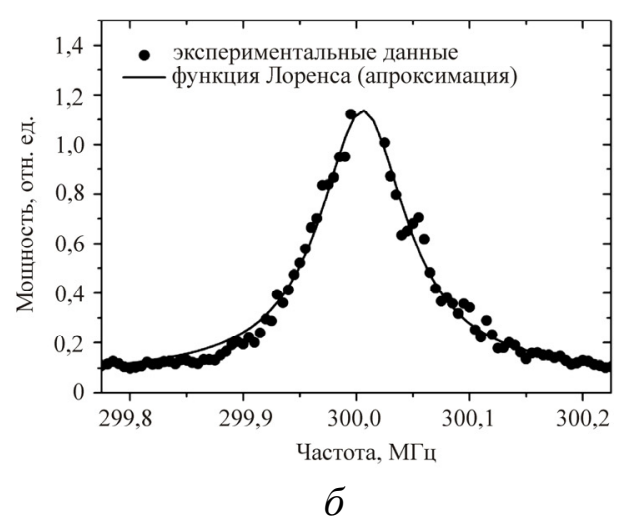

$\sigma$

Рис. 4. Спектры стоксовой компоненты $(a)$, распространяющейся назад, для конфигурации 1 (пунктирные линии) и конфигурации 2 (сплошные линии) при различных уровнях выходной мощности: 1 - порядка 1 мВт, 2 - 1,3 Вт, 3 - 3 Вт; спектр биений стоксовой компоненты (б), распространяющейся назад, для конфигурации 2 и аппроксимация функцией Лоренца 
Ширина линии в припороговом режиме была также измерена при помощи метода самогетеродинирования в схеме с интерферометром Маха-Цендера с улучшенным разрешением за счет многократного прохождения излучением закольцованного плеча интерферометра, включающего акустооптический модулятор (АОМ)[8]. В нашем случае длина закольцованного плеча составляла 1,5 км, что соответствует разрешающей способности $~ 100 / k$ кГц, где $k$ - номер обхода закольцованного плеча. Несущая частота АОМ составила 150 МГц. Ширина линии определялась по пику биений на удвоенной частоте сдвига $(k=2)$, задаваемого АОМ (300 кГц), для $k>2$ сигнал был слабый из-за больших потерь на сварках с АОМ. На рис. 4, $б$ представлен спектр биений, полученный с помощью радиочастотного анализатора Agilent Signal Analyzer N9010A с разрешением 10 кГц. Ширина сигнала биений, измеренная при мощности $\sim 10$ мВт, составила $\sim 100$ кГц (при разрешающей способности метода 50 кГц) (см. рис. 4, б), что соответствует ширине спектральной линии около 50 кГц и в 2 раза меньше ширины линии, полученной в [7] (100 кГц). Сужение линии в конфигурации 2, повидимому, связано с улучшением методики измерения ширины линии.

\section{Заключение}

В настоящей работе проведена оптимизация длины ВКР-лазера с распределенной обратной связью на основе массива коротких ВБР со случайными фазами и амплитудами [7]. Длина оптимизированного массива составила 6,7 м при общей длине волокна 7,7 м. Индикатором оптимизации являлось снижение уровня потерь на длине волны накачки и генерации. В результате оптимизации пороговая мощность накачки снизилась до 0,85 Вт по сравнению с полученной в [7], где она составила 1,3 Вт. Мощность генерации стоксовых компонент на длине волны 1092,4 нм составила в сумме около 6 Вт при мощности накачки 14 Вт. Ширина линии генерации в при пороговом режиме в данной конфигурации составила менее 50 кГц. При максимальной мощности накачки ширина линии не превышала 80 пм, что сравнимо с [7].

Создание ВКР-лазера представленного типа не требует специальных методик изготовления ВБР, при этом фазовые сдвиги имеют заведомо случайный характер, обусловленный преднамеренно низкой точностью (около 1 мм) выставления расстояния между ВБР при записи смежных решеток. Благодаря узкой спектральной ширине и достаточно большой мощности ВКР-лазер такого типа может использоваться 
в сенсорных системах. Также его излучение можно эффективно преобразовать в видимую область спектра за счет удвоения частоты в нелинейном кристалле или полингованном световоде. СРОС ВКР-лазер такого типа, реализованный в области 1,5 мкм, может применяться в телекоммуникациях.

Работа выполнена при поддержке Российского научного фонда (грант 14-22-0118). Авторы выражают благодарность за предоставленное оборудование ЦКП «Спектроскопия и оптика» (http://ckp-rf.ru/ ckp/3046/).

\section{Список литературы}

1. Random distributed feedback fibre laser / S.K. Turitsyn, S.A. Babin, A.E. El-Taher, P. Harper, D.V. Churkin, S.I. Kablukov, J.D. Ania-Castañón, V. Karalekas, E.V. Podivilov // Nature Photonics. - 2010. - Vol. 4, № 7. - P. 231-235.

2. Random distributed feedback fibre lasers / S.K. Turitsyn, S.A. Babin, D.V. Churkin, I.D. Vatnik, M.A. Nikulin, S.I. Kablukov, J.D. Ania-Castañón, V. Karalekas, E.V. Podivilov // Physics Reports. 2014. - Vol. 542, № 2. - P. 133-193.

3. Gagné M., Kashyap R. Demonstration of a $3 \mathrm{~mW}$ threshold Erdoped random fiber laser based on a unique fiber Bragg grating // Optics Express. - 2009. - Vol. 17, № 21. - P. 19067-19074.

4. Lizarraga N., Puente N.P., Chaikina E.I., Leskova T.A., Mendez E.R. Single-mode Er-doped fiber random laser with distributed Bragg grating feedback // Optics Express. - 2009. - Vol. 17, № 2. - P. 395-404.

5. Single-frequency Yb-doped fiber laser with distributed feedback based on a random FBG / S.R. Abdullina, A.A. Vlasov, I.A. Lobach, O. Belai, D.A. Shapiro, S.A. Babin // Laser Physics Letters. - 2016. Vol., № 7. - P. 075104.

6. Gagné M., Kashyap R. Random fiber Bragg grating Raman fiber laser // Optics Letters. - 2014. - Vol. 39, № 9. - P. 2755-2758.

7. Волоконный ВКР-лазер со случайной распределенной обратной связью на основе массива волоконных брэгговских решеток / М.И. Скворцов, С.Р. Абдуллина, А.А. Власов, Е.А. Злобина, И.А. Лобач, В.С. Терентьев, С.А. Бабин // Квантовая электроника. - 2017. T. 47, № 8. - C. 696-700. 
8. Tsuchida H. Simple technique for improving the resolution of the delayed self-heterodyne method // Optics Letters. - 1990. - Vol. 15, № 11. P. 640-642.

\section{References}

1. Turitsyn S.K., Babin S.A., El-Taher A.E., Harper P., Churkin D.V., Kablukov S.I., Ania-Castañón J.D., Karalekas V., Podivilov E.V. Random distributed feedback fibre laser. Nature Photonics, 2010, vol.4, no. 7, pp. 231-235.

2. Turitsyn S.K., Babin S.A., Churkin D.V., Vatnik I.D., Nikulin M.A., Kablukov S.I., Ania-Castañón J.D., Karalekas V., Podivilov E.V. Random distributed feedback fibre lasers. Physics Reports, 2014, vol. 542, no. 2, pp. 133-193.

3. Gagné M., Kashyap R. Demonstration of a $3 \mathrm{~mW}$ threshold Erdoped random fiber laser based on a unique fiber Bragg grating. Optics Express, 2009, vol. 17, no. 21, pp. 19067-19074.

4. Lizarraga N., Puente N.P., Chaikina E.I., Leskova T.A., Mendez E.R. Single-mode Er-doped fiber random laser with distributed Bragg grating feedback. Optics Express, 2009, vol. 17, no. 2, pp. 395-404.

5. Abdullina S.R., Vlasov A.A., Lobach I.A., Belai O., Shapiro D.A., Babin S.A. Single-frequency Yb-doped fiber laser with distributed feedback based on a random FBG. Laser Physics Letters, 2016, no. 7, pp. 075104.

6. Gagné M., Kashyap R. Random fiber Bragg grating Raman fiber laser. Optics Letters, 2014, vol. 39, no. 9, pp. 2755-2758.

7. Skvortsov M.I., Abdullina S.R., Vlasov A.A., Zlobina E.A., Lobach I.A., Terentev V.S., Babin S.A. Volokonnyy VKR-lazer so sluchaynoy raspredelennoy obratnoy svyazyu na osnove massiva volokonnykh breggovskikh reshetok [Fibrous Raman laser with random distributed feedback based on an array of fiber Bragg gratings]. Kvantovaya elektronika, 2017, vol. 47 , no. 8 , pp. 696-700.

8. Tsuchida H. Simple technique for improving the resolution of the delayed self-heterodyne method. Optics Letters, 1990, vol. 15, no. 11, pp. 640-642.

Получено 24.09.2018 